\title{
Das postthrombotische Syndrom - Häufigkeit, Diagnosestellung, Probleme bei der Klassifikation
}

\section{Post-thrombotic syndrome - prevalence, diagnosis, issues surrounding classification}

Autor

Tobias Hirsch

Institut

Praxis für Innere Medizin und Gefäßkrankheiten, Halle (Saale)

Schlüsselwörter

postthrombotisches Syndrom, Farbduplexsonografie, Scores

Key words

post-thrombotic syndrome, duplex ultrasound, scores

online publiziert 12.05 .2021

Bibliografie

Phlebologie 2021; 50: 176-183

DOI 10.1055/a-1427-0242

ISSN 0939-978X

(c) 2021. Thieme. All rights reserved.

Georg Thieme Verlag KG, Rüdigerstraße 14,

70469 Stuttgart, Germany

Korrespondenzadresse

Dr. med. Tobias Hirsch

Praxis für Innere Medizin und Gefäßkrankheiten, Halle (Saale)

Venen Kompetenz-Zentrum ${ }^{\circledR}$, Leipziger Straße 5,

06108 Halle (Saale), Deutschland

info@gefaessmedizin-hirsch.de

\section{ZUSAMMENFASSUNG}

Die Phlebothrombose und ihr chronischer Folgezustand, das postthrombotische Syndrom, besetzen in der Gefäßmedizin eine zentrale Position. Die Erkrankungen sind für die Betroffenen mit erheblichen Konsequenzen verbunden, die sich in vielen Fällen auf das gesamte weitere Leben auswirken. Obwohl das postthrombotische Syndrom eine Vielzahl typischer Symptome auslösen kann, ist die klinische Diagnosestellung nicht immer eindeutig und erfordert häufig zusätzliche technische Untersuchungsmethoden.

Die immer tiefergehenden Erkenntnisse über die Pathophysiologie einerseits und die Weiterentwicklung diagnostischer und therapeutischer Verfahren andererseits haben gerade in den letzten 20 Jahren zu einer erheblich stärkeren Sensitivität gegenüber der Erkrankung geführt und die Etablierung neuer Methoden vorangetrieben. So hat die Duplexsonografie die Phlebografie als Goldstandard in der Bildgebung abgelöst und Katheter-Verfahren haben einen festen Stellenwert neben oder sogar anstelle der offenen Chirurgie eingenommen.

Auch das Wissen über die seit Jahrzehnten etablierte Kompressionstherapie hat sich weiter vermehrt, sodass auch die konservativen Behandlungsstrategien einen Wandel erfahren haben.

Während für die Diagnostik und Therapie der Venenthrombose und Lungenembolie Leitlinien vorliegen, existieren keine aktuellen Empfehlungen, die die modernen Entwicklungen für das postthrombotische Syndrom berücksichtigen.

Der Übersichtsartikel befasst sich mit dem diagnostischen Vorgehen unter der besonderen Berücksichtigung der Duplexsonografie und ordnet die weiteren zur Verfügung stehenden bildgebenden und funktionsdiagnostischen Methoden ein.

\section{ABSTRACT}

Phlebothrombosis and its sequelae, post-thrombotic syndrome, play a leading role in vascular medicine. The diseases have considerable consequences for those affected which, in many cases, could last for the rest of their lives. Although post-thrombotic syndrome can trigger a variety of typical symptoms, clinical diagnosis is not always straightforward and often requires additional technical methods of examination.

Awareness of the disease has grown considerably, especially in the last 20 years, due in part to better knowledge of the pathophysiology and the further development of diagnostic and therapeutic tools. This has driven forward the establishment of new methods. Duplex ultrasound, for example, has replaced phlebography as the gold standard in imaging, and catheter procedures have become firmly established alongside or even in place of open surgery. Knowledge about compression therapy, which has been an established treatment method for decades, has also continued to increase, reflecting the fact that conservative treatment strategies have also undergone a transformation.

While guidelines have been established for the diagnosis and treatment of venous thrombosis and pulmonary embolism, there are currently no recommendations that take into account the modern developments for post-thrombotic syndrome.

This review article looks at the diagnostic process, paying particular attention to duplex ultrasound. It also classifies the other available imaging and functional diagnostic methods. 


\section{Einleitung}

Die tiefe Venenthrombose (TVT) stellt wegen ihrer prognostischen Bedeutung ein zentrales Thema in der Phlebologie dar. Einerseits kann sie die Ursache für eine akut lebensbedrohliche Lungenarterienembolie darstellen. Andererseits kann sie als Folgezustand eine chronische venöse Insuffizienz (CVI) hinterlassen, die in diesem Falle als postthrombotisches Syndrom (PTS) bezeichnet wird.

Die internationale Klassifikation der Krankheiten (ICD-10) verschlüsselt die Diagnose „postthrombotisches Syndrom“ unter der Ziffer 187.0. Diese eindeutige Zuordnung suggeriert, dass es sich um ein definiertes Krankheitsbild handelt. Ein Syndrom ist laut Duden ein „Krankheitsbild, das sich aus dem Zusammentreffen verschiedener charakteristischer Symptome ergibt“. Auf der Website der Deutschen Gesellschaft für Phlebologie wird die Erkrankung beschrieben als „Folgeerscheinungen, die Monate bis Jahre nach einer tiefen Venenthrombose“ auftreten. Die etymologischen Erklärungen lassen bereits eine ausgeprägte Unschärfe bei der Begriffsdefinition erkennen.

Die CEAP-Klassifikation lässt sich auf das PTS anwenden und liefert eine Charakterisierung des Krankheitsbildes. Neben dem klinischen Stadium, das auch beim PTS vom leichten, expositionell verstärkten Schweregefühl bis zum Ulcus cruris ausgeprägt sein kann, sind die weiteren Buchstaben des Akronyms relevant: ESi besagt, dass es sich ätiologisch um ein sekundäres Krankheitsbild mit intravenöser Ursache handelt. Da Veränderungen am tiefen Venensystem verantwortlich sind, findet zur Anatomie AD Verwendung. Als pathologisches Korrelat können sowohl Obstruktionen als auch Reflux eine Rolle spielen, sodass eine Beschreibung sowohl mit PR, PO oder PR,O denkbar wäre [1]. Unter Anwendung dieser Klassifikation lässt z. B. die Einteilung C4 ESi AD PR, $\mathrm{O}_{\text {VIE }}$ darauf schließen, dass Hautveränderungen am Bein Folge eines PTS nach Thrombose der V. iliaca externa mit Obstruktion und zusätzlichem Reflux aufgrund postthrombotischer Klappendestruktion sind.

Wenngleich diese überarbeitete Version der CEAP-Klassifikation die Beschreibung der Krankheit erleichtert, ergeben sich in der Praxis dennoch mitunter Schwierigkeiten sowohl bei der pathophysiologischen Einordnung und der Therapieplanung als auch in der gutachterlichen Bewertung.

\section{Epidemiologie des postthrombotischen Syndroms}

Beim postthrombotischen Syndrom handelt es sich um ein chronisches Krankheitsbild mit lang beobachtbarem Verlauf. Es existieren vergleichsweise viele Daten zur Inzidenz und zur Prävalenz von Phlebothrombosen und PTS. Verschiedene Studien schätzen die Inzidenz venöser Thromboembolien auf 0,2-2 pro $1000 \mathrm{~Pa}$ tienten/Jahr [2, 3].

Eine multidisziplinäre Expertengruppe unter Leitung von Susan Kahn erstellte 2014 im Auftrag der American Heart Association ein Übersichtspapier zum postthrombotischen Syndrom. In der Arbeit wird die Inzidenz der TVT in weitgehender Übereinstimmung zu den o.g. Daten mit 1-3 von 1000 Menschen pro Jahr in der Gesamtbevölkerung angegeben. Die Entwicklung eines postthrombotischen Syndroms wird für $20-50 \%$ der Patienten erwartet [4].

Die Prävalenz des PTS wurde in der Bonner Venenstudie I mit $1,1 \%$ ermittelt [5]. Prandoni et al. beziffern die Prävalenz mit 1$5 \%[6]$.

Ein schwergradiges PTS wird laut Kahn bei 5-10\% der Betroffenen beobachtet [7].

Eine amerikanische Arbeitsgruppe verglich 2006 die Kosten, die entstehen, wenn sich nach einer Phlebothrombose ein PTS entwickelt, mit denen, wenn es gelingt, ein PTS zu verhindern. In der PTS-Gruppe standen durchschnittliche jährliche Aufwendungen von \$20 569 den Kosten in Höhe von \$15843 gegenüber, wenn sich kein PTS entwickelt, was auf vermehrte Arztbesuche, technische Untersuchungskosten und Kosten für Heil- und Hilfsmittel und Medikamente zurückzuführen ist. Wenn man diese Differenz auf die o.g. epidemiologischen Daten bezieht und berücksichtigt, dass das PTS einen chronischen und nicht selten progredienten Verlauf nehmen kann, lässt sich ableiten, welche immense gesundheitsökonomische Bedeutung die Erkrankung hat [8].

\section{Diagnosestellung}

Die Diagnose eines postthrombotischen Syndroms lässt sich bei den meisten Patienten allein anhand des klinischen Befundes und der Anamnese stellen. Die Patienten können sich mit sämtlichen möglichen Symptomen einer CVI vorstellen, vom Schweregefühl in der betroffenen Extremität im Tagesverlauf bis hin zu erheblichen Hautveränderungen und Exulzerationen. Meist können auch Angaben zu einer stattgehabten Thrombose in der Krankengeschichte gemacht werden.

Laut American Venous Forum besteht ein postthrombotisches Syndrom, wenn die folgenden 4 Kriterien erfüllt sind: das tägliche Auftreten von Schwellung und Schmerzen im Bein seit mindestens einem Monat, der Beginn dieser Beschwerden 6 Monate oder länger nach einer tiefen Beinvenenthrombose, Verschlechterung der Symptome beim Stehen oder Laufen, Verbesserung bei Ruhe und Hochlagerung der Extremität („Ginsberg measure“ [9]). Diese Definition besitzt zwar den Charme, dass sie sich leicht im Rahmen wissenschaftlicher Untersuchungen anwenden lässt. Allerdings wird sie der Komplexität des Krankheitsbildes nicht gerecht, da z. B. auf Hautveränderungen gar nicht eingegangen wird.

Es existiert eine Reihe von Fallstricken, die eine weiterführende Diagnostik erforderlich machen können. So muss einerseits nicht jede in der Anamnese explorierte Phlebothrombose zur Ausprägung eines PTS führen, wohingegen aber typische Symptome eines Venenleidens auch durch andere Ursachen imitiert werden können. Von besonderer Bedeutung sind Komorbiditäten wie Adipositas, Lymphödem, radikuläre Blockierungen, aber auch Hautaffektionen wie Kontaktekzeme und Kratzaffekte und nicht zuletzt die primäre Varikose, die differenzialdiagnostisch abgegrenzt werden müssen.

Andererseits kann eine ursächliche Thrombose unerkannt sein, was insbesondere in der Traumatologie eine Rolle spielt, da 
Schwellung und Schmerzen in der betroffenen Extremität auch charakteristische Symptome z. B. einer Fraktur etc. sind. Nicht selten wird ein PTS erst Jahre oder sogar Jahrzehnte nach einem Trauma im Zusammenhang mit einer Begutachtung diagnostiziert.

Die Farbdoppler- und Duplexsonografie als Standardmethode ermöglicht die weitere Beurteilung des postthrombotischen Syndroms und auch die Differenzierung von einer frischen Phlebothrombose.

Die aszendierende Press-Phlebografie hat als Primärdiagnostikum keine Bedeutung mehr. Eine radiologische Bildgebung wird so gut wie ausschließlich im Rahmen geplanter Interventionen eingesetzt, wie auch Schnittbildverfahren und der intravenöse Ultraschall (IVUS), weshalb in dieser Arbeit nicht näher auf diese Methoden eingegangen werden soll.

Phlebologische Funktionsdiagnostik wie venöse VerschlussPlethysmografie (VVP), Lichtreflex-Rheografie (LRR) oder Phlebodynamometrie (PDM) kann in Einzelfällen zusätzliche behandlungsrelevante Informationen liefern und spielt vor allem in der Verlaufskontrolle und der Begutachtung eine Rolle.

\section{Klinischer Befund und Krankheitsverlauf}

Zum Verständnis der Pathophysiologie ist die von Hach und HachWunderle eingeführte Untergliederung in 3 Erkrankungsstadien didaktisch sinnvoll. Als postthrombotisches Frühsyndrom bezeichnen die Autoren die Phase der Rekanalisierung und Kollateralisierung in den ersten Wochen nach der Phlebothrombose, die vor allem von einer Ödembildung geprägt ist. Dem schließt sich das postthrombotische Syndrom (im engeren Sinne) an, welches über viele Jahre stabil sein kann und durch die o. g. Ginsbergsche Symptom-Tetralogie gekennzeichnet ist. Nach Jahren bis Jahrzehnten schließlich entwickelt sich als Ausdruck einer Dekompensation der Kollateralvenen das postthrombotische Spätsyndrom. In dieser Phase lassen sich häufig eine Perforansinsuffizienz und eine sekundäre Stammvarikose feststellen. Im klinischen Erscheinungsbild können Hautveränderungen bis hin zum Ulcus cruris hinzutreten [10]. An dieser Stelle sei nur erwähnt, dass zusätzlich komplexe inflammatorische Vorgänge an der Pathogenese beteiligt sind.

Der Krankheitsverlauf kann individuell sehr unterschiedlich verlaufen. Maßgebliche Einflussfaktoren sind das Alter, die betroffene Etage und vor allem das Auftreten von Rezidivthrombosen.

Die Unterscheidung dieser 3 Krankheitsphasen ist sinnvoll, da die Kenntnis der pathophysiologischen Entwicklung die Interpretation der klinischen Befunde, der Ultraschallbefunde und der phlebologischen Funktionsdiagnostik erleichtert.

\section{Die Duplexsonografie markiert den Goldstandard der Diagnostik}

Die Ultraschalldiagnostik liefert Informationen zur Lokalisation des PTS und zum Grad von Rekanalisierung und Kollateralisierung. Eine Korrelation zwischen Ultraschallbefund und Klinik ist dabei nicht verlässlich zu erwarten, sodass bisweilen beeindruckende
Ultraschallbefunde mit nur geringer Symptomatik einhergehen können und gleichzeitig massive Hautbefunde nur geringfügige Veränderungen im Ultraschall aufweisen.

Über die topografische Manifestation postthrombotischer Veränderungen existieren nur wenige Daten. Eine italienische Arbeitsgruppe detektierte unter 189 Patienten mit tiefer Beinvenenthrombose an 166 eine proximale und nur an 23 eine distale Thrombose. Eine isolierte Thrombose der Beckenvenen wurde in der untersuchten Gruppe nicht nachgewiesen [11]. Johnson et al. führten über 6 Jahre Nachuntersuchungen an 83 Beinen durch, an denen eine tiefvenöse Thrombose aufgetreten war. 23 zeigten ein Ödem, 11 eine Hyperpigmentierung, 2 wiesen ein Ulcus cruris auf. $65 \%$ hatten sowohl eine Obstruktion als auch einen Reflux entwickelt. Während 59 \% $(n=49)$ keine Symptome eines PTS aufwiesen, war in der Ultraschalluntersuchung nur in $12 \%$ eine vollständige Normalisierung festzustellen [12]. Dies kann exemplarisch als Beleg dafür angesehen werden, wie stark klinischer und Ultraschallbefund voneinander abweichen können.

In weiteren vergleichenden Ultraschalluntersuchungen an Patienten mit und ohne PTS nach tiefer Venenthrombose konnte dieselbe Arbeitsgruppe belegen, dass ein klinisch relevantes PTS signifikant häufiger assoziiert war mit postthrombotischen Veränderungen in der Vena poplitea und den Venae tibiales posteriores, wohingegen sich Veränderungen auf der Etage der Magnacrosse in den beiden Gruppen nicht signifikant unterschieden [13]. Dieser Umstand entspricht der Beobachtung des Autors in der täglichen Praxis und ist insofern bemerkenswert, da der einzige aktuell verfügbare und verstärkt im wissenschaftlichen Fokus stehende interventionelle Behandlungsansatz gerade nur auf diese Gefäßetage (Beckenvenen) abzielt.

Die Funktion der Vena profunda femoris ist wenig untersucht, wenngleich das Gefäß in der Venenchirurgie durchaus eine Rolle spielt. Der duplexsonografischen Exploration entzieht sie sich weitgehend bzw. ist lediglich über wenige Zentimeter im Bereich der Gabel darstellbar.

Die Bedeutung der Duplexsonografie bei der Behandlung des PTS liegt neben dem qualitativen Nachweis eines PTS im Sinne der Differenzialdiagnose in der Abgrenzung von einer akuten Phlebothrombose und der Befunddokumentation im Rahmen der Verlaufskontrolle. Insbesondere der letztgenannte Punkt ist in der Praxis von großer Relevanz, da von einer Befunddynamik, etwa in der Folge rezidivierender Thrombosen, das Antikoagulationsmanagement abhängen kann.

\section{Typische Ultraschallbefunde des postthrombotischen Syndroms}

In der Frühphase des postthrombotischen Syndroms unterscheidet sich der duplexsonografische Befund nur wenig von dem der akuten Phlebothrombose. Eine Kollateralisierung ist oft noch nicht ausgebildet und die betroffenen Venen sind subtotal oder komplett okkludiert ( $\triangleright$ Abb. 1a, b).

Im Verlauf der Vena femoralis kann mitunter ihre Doppelanlage an Bedeutung gewinnen ( $\triangleright$ Abb. $2 \mathbf{a}-\mathbf{c}$ ).

Eine Rekanalisierung kann im Verlauf zu beobachten sein. In den ersten Wochen ist der Thrombus reflexarm und homogen, 

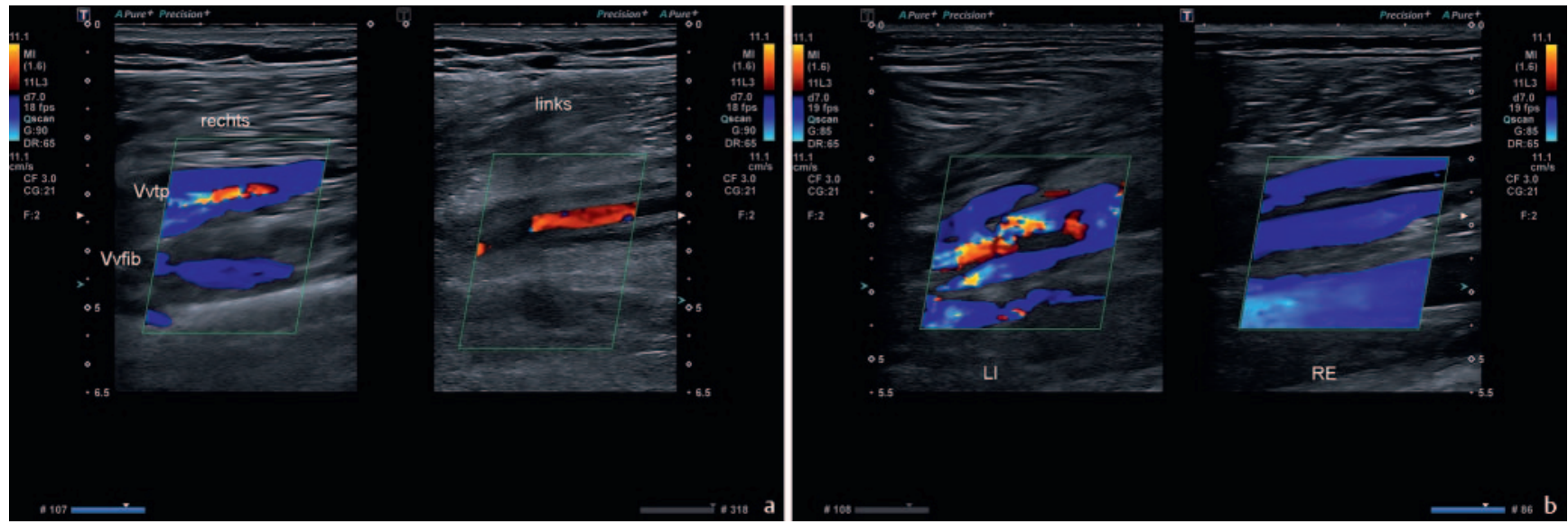

- Abb. 1 Akute Thrombose vs. postthrombotisches Syndrom. a Akute Phlebothrombose der Vv. tibiales posteriores links. Die Venen sind komplett reflexreich ausgefüllt ohne Flusssignal wie in der gesunden Gegenseite. b PTS der Vv. tibiales posteriores links. Gegenüber dem homogenen Farbsignal auf der Gegenseite zeigt sich ein inhomogenes, aufgrund von Turbulenzen „buntes“ Farbbild mit Aliasing und Kollateralen.
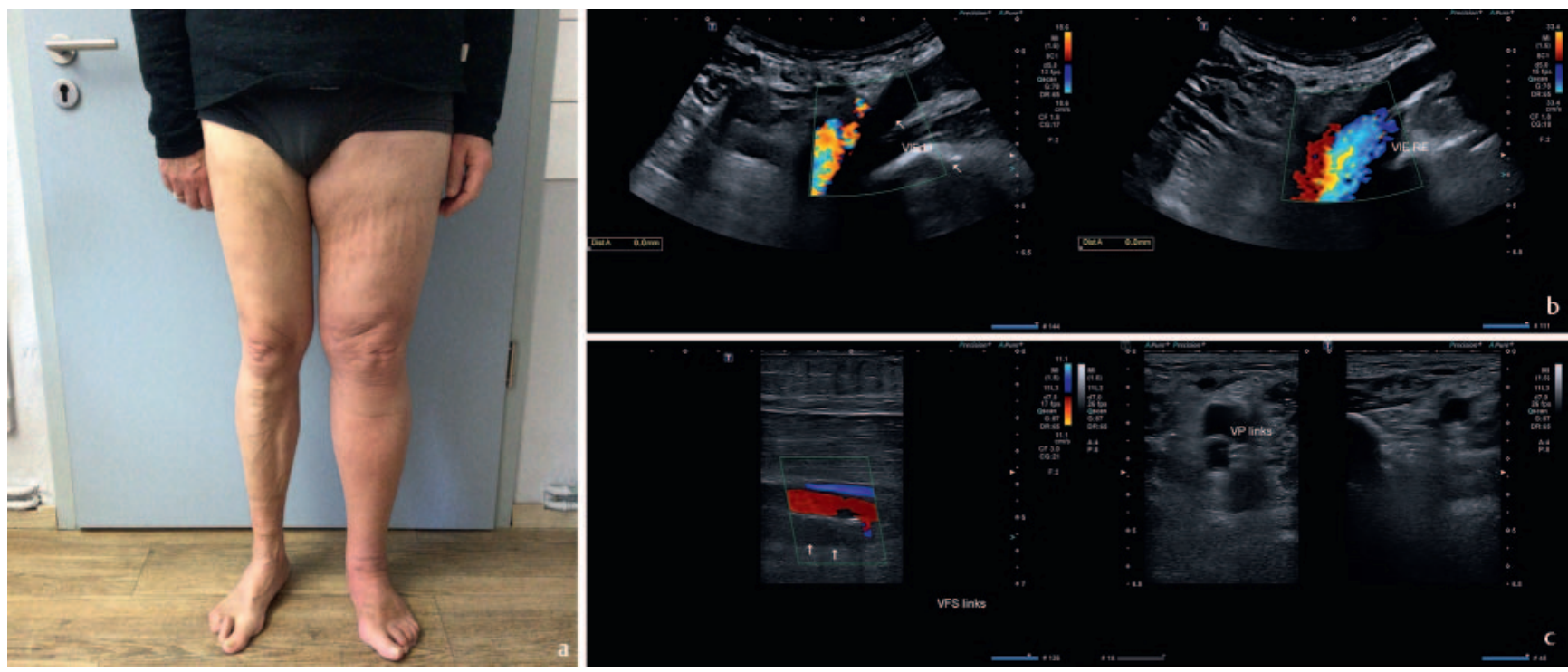

- Abb. 2 Postthrombotisches Frühsyndrom. a Klinischer Befund bei 14 Tage alter Beckenvenenthrombose des linken Beins: ausgeprägtes perimalleoläres und crurales Ödem, Umfangsdifferenz bis $11 \mathrm{~cm}$. b Fortbestehende komplette Okklusion der V. iliaca externa und V. iliaca communis links. Die Darstellung dieser Region erfordert den Einsatz der Konvexsonde. Es empfiehlt sich der Seitenvergleich. c Die V. femoralis links ist noch komplett okkludiert. Als Ausdruck der Kompensation ist bereits die ventral der Arterie darstellbare Doppelanlage der Vene rekrutiert. Die V. poplitea ist in der Kompressionssonografie thrombusfrei (rechtes Bild).

was dafür spricht, dass er noch nicht fibrotisch organisiert ist. Diese Krankheitsphase zu erkennen ist von therapeutischer Relevanz, da zu diesem Zeitpunkt sowohl die Kompressionstherapie konsequent appliziert werden muss, da sie noch einen Einfluss auf die weitere Entwicklung des PTS hat [14], und in dieser Phase auch interventionelle und chirurgische Akutmaßnahmen noch sinnvoll sein können.

Das postthrombotische Syndrom im eigentlichen Sinne ist gekennzeichnet durch einen zunehmend strähnigen Umbau im Sinne der Fibrose bei gleichzeitiger Rekanalisierung und Ausbildung von Kollateralen. Für den klinischen Befund besitzt jedoch vor allem der Reflux aufgrund der destruierten Venenklappen eine Bedeutung. Während die Darstellung der Leitvenen ab Knie-Etage aufwärts in der Regel leichtfällt, kann die aussagekräftige Bildgebung im Unterschenkelbereich Probleme bereiten, da Ödem, Hautindurationen oder auch Unterhautfett die in der Tiefe der Muskulatur verlaufenden Venenpaare maskieren können. Es empfiehlt sich aus diesem Grunde, stets den Vergleich mit der gesunden Seite zu suchen ( $\bullet \mathbf{A b b}$. 3a-c). Bei besonders kräftigen Beinen kann der Einsatz der Konvexsonde ebenso sinnvoll sein wie im Beckenbereich.

Die beschriebenen Veränderungen lassen sich auch beim postthrombotischen Spätsyndrom nachweisen. In dieser Phase, die aufgrund der sehr langen Krankengeschichte mit einer weiteren Verschlechterung des Hautbildes einhergeht, lässt sich als Ausdruck der Überlastung kompensatorischer Mechanismen eine zu- 

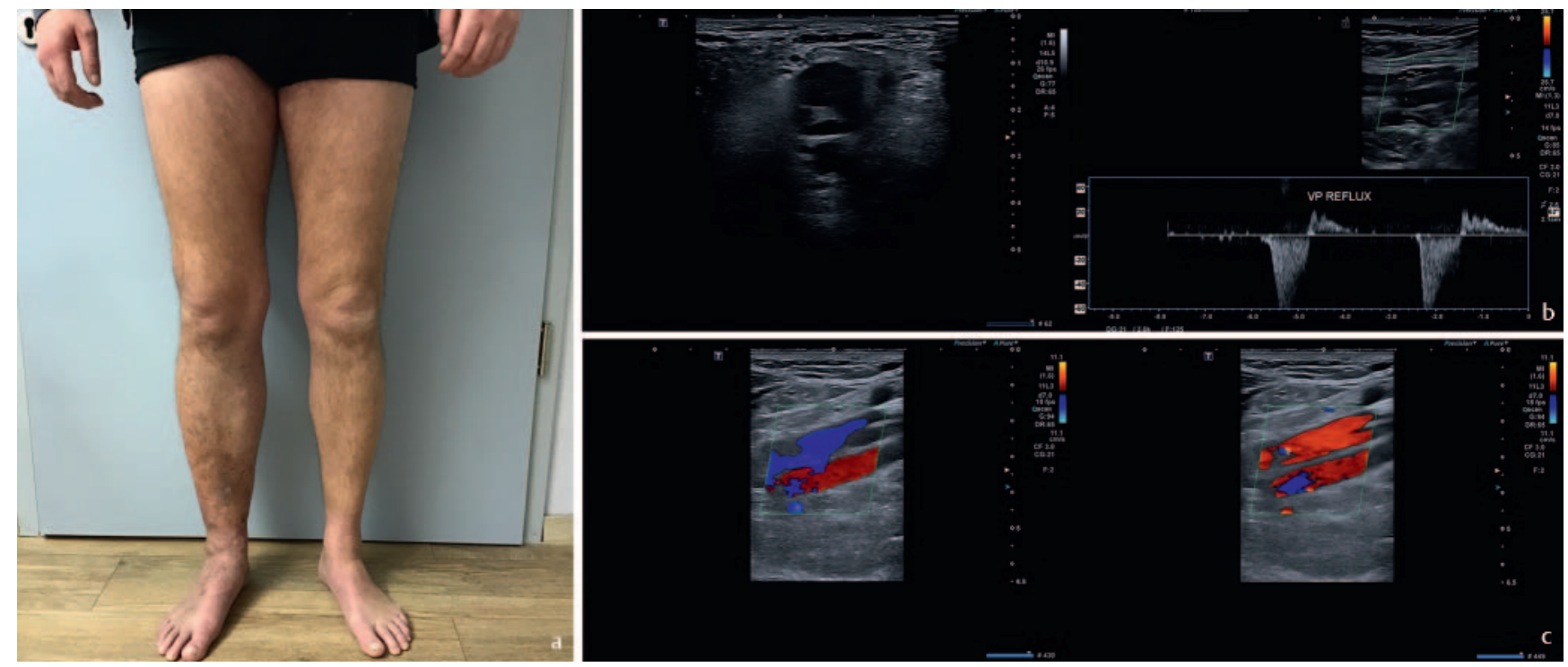

- Abb. 3 Postthrombotisches Syndrom im eigentlichen Sinne. a Klinischer Befund bei PTS 12 Jahre nach 3-Etagen-Thrombose des rechten Beins: umschriebenes Knöchelödem (Schwellneigung im Tagesverlauf), deutliche Hyperpigmentierung als Ausdruck der trophischen Hautveränderungen, kleine Ulkusnarbe am Schienbein. b „Pseudoseptenartige“ Residualbefunde in der V. poplitea rechts bei partieller Rekanalisierung und Leitvenenreflux im PW-Doppler (rechtes Bild). c Im Provokationsmanöver stellt sich die V. poplitea aufgrund der postthrombotischen Residuen mit inkompletter Farbfüllung dar, in der Muskeldiastole deutlicher Farbumschlag als Ausdruck des tiefvenösen Refluxes (rechts).

sätzliche Insuffizienz der epi- und transfaszialen Venen beobachten: Perforatoren werden insuffizient und nehmen im Durchmesser zu und eine Stammvarikose verstärkt sich oder entsteht. Der daraus resultierende zusätzliche Reflux führt zu einer Erhöhung des ambulatorischen Venendrucks mit konsekutiver Verstärkung der Symptome oder sogar Verschlechterung der trophischen Situation der Haut. Häufig zu beobachten ist dieser Prozess im Bereich der Perforatoren der Tibialis-posterior-Gruppe (frühere Nomenklatur: Cockett-Gruppe). Sie verbinden die hintere Bogenvene oder Äste der V. saphena parva mit den Vv. tibiales posteriores. Im Normalfall drainieren sie das Blut aus den epifaszialen Gefäßen in die tiefen Beinvenen, sodass es sich nicht zwingend um einen pathologischen Reflux handelt, sondern um den physiologischen Wiedereintritt in das tiefe Venensystem. Eine echte Perforansinsuffizienz hingegen entwickelt sich im Rahmen eines PTS, wenn der reguläre Abstrom über die $\mathrm{Vv}$. tibiales posteriores aufgrund einer Okklusion oder eines Leitvenenrefluxes gestört ist. In dem Fall kann eine deutliche sekundäre Varikose distal der Cockett-Gruppe erkennbar werden, die zu einem Ödem im Bereich des Innenknöchels, einer Corona phlebectatica oder zur Verstärkung von Hautveränderungen führt ( $\triangleright \mathbf{A b b} \mathbf{4} \mathbf{4 a}, \mathbf{b})$.

Wenngleich sich im Laufe der Jahre die Entwicklung eines postthrombotischen Syndroms von den Frühsymptomen zum Spätsyndrom in der Regel verfolgen lässt, so bedeutet dies nicht, dass ein postthrombotisches Spätsyndrom auch regelhaft mit massiven Hautveränderungen und Beschwerden einhergehen muss. Zum einen hängt diese Entwicklung vom Ausmaß der zugrunde liegenden Thrombose und von einer Reihe begleitender individueller Faktoren ab (Alter, Gewicht, Komorbiditäten), zum anderen ist gerade diese Entwicklung durch eine adäquate Therapie auch zu beeinflussen.

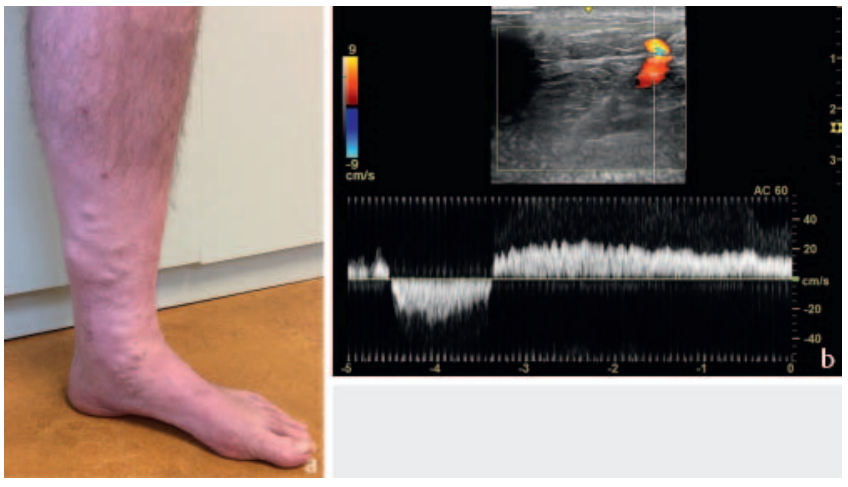

- Abb. 4 Postthrombotisches Spätsyndrom. a Symptomatische Varikose im Bereich der rechten Innenknöchels 6 Jahre nach Fraktur des oberen Sprunggelenks. Ein postthrombotisches Syndrom der Vv. tibiales posteriores war bis zum Untersuchungszeitpunkt nicht bekannt. b Der insuffiziente Perforator der Tibialis-posterior-Gruppe stellt den proximalen Insuffizienzpunkt dar. Der physiologische Wiedereintritt und Abstrom sind aufgrund der postthrombotischen Veränderungen in den tiefen Venen gestört ( $V$. saphena magna ist kompetent).

\section{Die phlebologische Funktionsdiagnostik ergänzt die Befunddokumentation}

Aufgrund ihrer exzellenten optischen Auflösung und der Fülle an dynamischen Informationen, welche moderne Ultraschallmaschinen liefern, sind unter Kenntnis von Krankengeschichte und klinischem Befund in der Regel weitere bildgebende oder diagnostische Verfahren nicht erforderlich, um ein postthrombotisches Syndrom zu erkennen bzw. adäquat zu behandeln. 


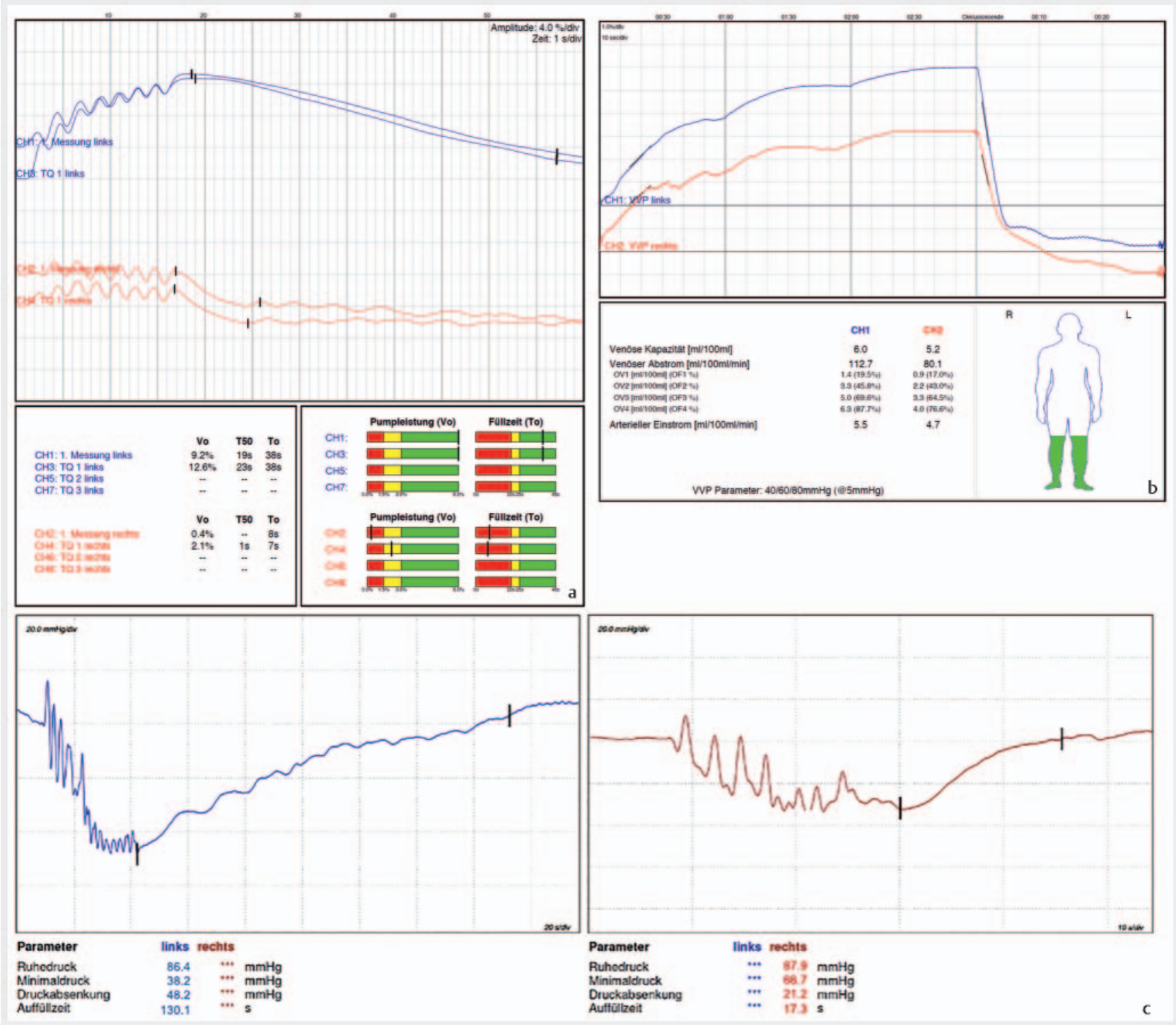

- Abb. 5 Phlebologische Funktionsdiagnostik: LRR und VVP (Patient aus $>$ Abb. 3). a Lichtreflex-Rheografie (LRR): Gestörte Pumpleistung im Arbeitsmanöver und im Seitenvergleich deutliche raschere venöse Wiederauffüllzeit rechts ( $8 \mathrm{~s}$ vs. $38 \mathrm{~s}$ ) belegen den venösen Reflux. $\mathbf{b}$ Venöse Verschluss-Plethysmografie VVP): Im Seitenvergleich verlangsamter venöser Abstrom am erkrankten rechten Bein (80,1 ml/min vs. $112,7 \mathrm{ml} / \mathrm{min})$ als Ausdruck der obstruktiven Komponente des PTS. c Phlebodynamometrie (PDM): Die Absenkung des Venendrucks ist im erkrankten rechten Bein erheblich reduziert gegenüber dem gesunden $(21,2 \mathrm{mmHg}$ vs. $48,2 \mathrm{mmHg}$ ). Darüber hinaus ebenfalls beschleunigte venöse Auffüllzeit (17,3 s vs. 130,1 s, beachte die geänderte Zeitachse).

Die Einbeziehung der traditionellen phlebologischen Verfahren VVP, LRR und PDM kann sich besonders dann als nützlich erweisen, wenn konkurrierende Krankheitszustände in ihrer pathologischen Bedeutung gegeneinander abgewogen werden müssen, wie z. B. ein vorrangig okklusives PTS bei gleichzeitigem Vorliegen eines Refluxes im Rahmen einer primären Stammvarikose $(\triangleright$ Abb. 5a-c).

VVP und LRR sind aufgrund ihrer unkomplizierten und schnellen Durchführung auch hervorragend zur Dokumentation des Krankheitsverlaufs geeignet. So kann beispielsweise eine plötzliche Verschlechterung des venösen Abstroms in der VVP die Differenzierung eines PTS mit frisch stattgehabter Rezidiv-Thrombose von einem älteren postthrombotischen Residualbefund erleichtern. Gerade diese Differenzialdiagnostik ist oftmals allein mit Ultraschall nicht möglich.

Die Methoden haben darüber hinaus noch im Rahmen der medizinischen Begutachtung einen Stellenwert. Thrombose und postthrombotisches Syndrom sind nicht selten Anlass für Begutachtungen im Rahmen des Entschädigungsrechts der gesetzlichen Unfallversicherung.

Da sich ein postthrombotisches Syndrom über einen langen Zeitraum entwickelt, in welchem naturgemäß auch eine Reihe von Komorbiditäten eintreten können, kann es für einen Gutachter nützlich sein, wenn er seine Argumentation durch Messwerte 
belegen kann. Ein schwergradiges PTS als Unfallfolge kann eine entschädigungspflichtige Minderung der Erwerbsfähigkeit (MdE) bis zu 50 \% begründen [15].

Am besten belastbar ist die direkte Messung des Venendrucks mittels Phlebodynamometrie. Jedoch ist die Methode aufgrund ihrer Invasivität und des Untersuchungsaufwandes nicht als Screening-Methode geeignet. Die Nachteile von Lichtreflex-Rheografie und venöser Verschluss-Plethysmografie bestehen in ihrer Anfälligkeit gegenüber äußeren Fakten wie Raumtemperatur, Tageszeit und Hautzustand. Aus diesem Grunde sollte die Bewertung ihrer Ergebnisse nicht in erster Linie auf der Basis der gelieferten Absolutwerte erfolgen, sondern stets unter Berücksichtigung der Werte der gesunden Extremität.

\section{Die Klassifikation des postthrombotischen Syndroms mithilfe von Scores}

Zur wissenschaftlichen Analyse sowie im Rahmen der gutachterlichen Bewertung ist immer wieder versucht worden, das PTS zu quantifizieren. Eine niederländische Arbeit hat verschiedene Definitionen des Krankheitsbildes und international übliche Score-Systeme evaluiert und dabei insbesondere auch deren Korrelation mit dem maßgeblichen pathologischen Faktor, dem ambulatorischen Venendruck, untersucht. Kolbach et al. analysierten die Widmer- und die CEAP-Klassifikation, den Venous-Clinical-Severity-Score (VCSS) und die Scores von Villalta und Brandjes. Die Autoren kamen dabei zu dem Ergebnis, dass alle Scores in unterschiedlicher Weise subjektive Beschwerden und objektivierbare Symptome berücksichtigen und eine Korrelation zur venösen Hypertension aufweisen, wobei letztere allerdings nur in den schweren Krankheitsstadien relevant sind [16].

Die Widmer-Klassifikation beschränkt sich in 3 Schweregraden lediglich auf die klinischen Symptome. Sie kann als verlassen betrachtet werden, da sie durch die 2020 neu überarbeitete CEAPKlassifikation abgelöst wurde, die, wie eingangs beschrieben, neben der konkreteren Beschreibung des klinischen Zustandes auch Informationen über die Anatomie, die Ätiologie und die Pathophysiologie liefert.

Der Venous Clinical Severity Score (VCSS) erweitert die klinische Klassifikation um eine Schweregradeinteilung (0-3). Zusätzlich werden auch das Symptom Schmerz und die Ulkusgröße erfasst sowie die Intensität der erforderlichen Kompressionstherapie. Der Score ist für das PTS ebenso gut anwendbar wie für die Varikose und ist ein Standardwerkzeug in Studien [17]. Der Villalta-Score (nach dem Hauptautor der bekannteren, obgleich später erschienenen Publikation auch Prandoni-Score genannt) erfasst neben den klinischen Symptomen zusätzlich die subjektiven Beschwerden des Patienten ( $\triangleright$ Tab. 1) [18]. Er ist übersichtlich aufgebaut und damit nicht nur für Studien geeignet, sondern sehr alltagstauglich auch in der täglichen Praxis.

Für jede Qualität können 0-3 Punkte vergeben werden (nicht gering - moderat - schwer). Ein Score von 5-14 entspricht einem milden bis moderaten PTS. Ein Score von $\geq 15$ bzw. inkl. Ulcus cruris entspricht einem schwergradigen PTS.

Im Rahmen einer Studie zur Messung des Effektes der Kompressionstherapie bei Patienten mit symptomatischen proximalen
Tab. 1 Villalta-Score [18]

\begin{tabular}{|l|l|l|l|l|}
\hline Schweregrad & $\mathbf{0}$ & $\mathbf{1}$ & $\mathbf{2}$ & $\mathbf{3}$ \\
\hline Beschwerden & & & \\
\hline - Schmerz & & \\
\hline - Schweregefühl & & \\
\hline - Krämpfe & & \\
\hline - Juckreiz & & \\
\hline - Parästhesien & & \\
\hline klinische Zeichen & & \\
\hline - prätibiales Ödem & & \\
\hline - Hautindurationen & & \\
\hline - Hyperpigmentierung & & \\
\hline - neue Venenektasien & & \\
\hline - Rötung & & \\
\hline - Wadendruckschmerz & & \\
\hline - Ulcus cruris & & \\
\hline
\end{tabular}

Beinvenenthrombosen, die 1997 im Lancet erschien, wurde der Brandjes-Score entwickelt [19]. Er erfasst neben objektivierbaren Kriterien wie Beinumfängen und Hautveränderungen auch subjektiv angegebene Symptome wie Spontanschmerz bzw. durch Stehen oder Laufen provozierten Schmerz, Schweregefühl und Einschränkungen bei der Verrichtung von Alltagsaktivitäten. Der relativ aufwendige Score zielte darauf ab, ein mildes bis moderates PTS von der schweren Verlaufsform zu unterscheiden.

Die Scores berücksichtigen in unterschiedlicher Weise subjektive Beschwerden und objektivierbare klinische Symptome. Es besteht eine Korrelation zum Venendruck, wobei dieser bei schwergradigen Krankheitsstadien deutlicher erkennbar ist als bei milden bis moderaten Stadien des PTS. Jedes der verschiedenen, in der wissenschaftlichen Literatur gebräuchlichen Score-Systeme besitzt seine eigene Charakteristik, seine Schwerpunkte, aber auch Lücken, ohne dass einer der Scores Überlegenheit aufweist. Zur wissenschaftlichen Betrachtung des PTS sollten daher möglichst verschiedene Scores Verwendung finden, während nach Ansicht des Autors die Implementierung eines der Scores in der praktischen Routine zu empfehlen ist.

\section{Fazit}

1. Das postthrombotische Syndrom besitzt neben der individuellen Bedeutung für den betroffenen Patienten eine große gesundheitsökonomische Bedeutung.

2. Neben der Zuordnung der typischen klinischen Befunde eines postthrombotischen Syndroms und der Anamneseerhebung ist die Duplexsonografie die Methode der ersten Wahl zur Sicherung und Beurteilung des postthrombotischen Syndroms.

3. Die verlässliche Quantifizierung des Krankheitsbildes ist schwierig. Im Rahmen wissenschaftlicher Untersuchungen und im Zusammenhang mit der Begutachtung stellen evaluierte 
Scores und Klassifikationen ein nützliches Werkzeug dar. Ihre Verwendung sollte in der deutschen Phlebologie gestärkt werden.

\section{Interessenkonflikt}

Die Autorinnen/Autoren geben an, dass kein Interessenkonflikt besteht.

Literatur

[1] Lurie F, Passman M, Meissner M et al. The 2020 update of the CEAP classification system and reporting standards. J Vasc Surg Venous Lymphat Disord 2020; 8 (3): 342-352. doi:10.1016/j.jvsv.2019.12.075. Epub 2020 Feb 27. Erratum in: J Vasc Surg Venous Lymphat Disord 2021 Jan; 9(1): 288. PMID: 32113854

[2] Spencer FA, Emery C, Joffe SW et al. Incidence rates, clinical profile, and outcomes of patients with venous thromboembolism: The Worcester VTE study. J Thromb Thrombolysis 2009; 28: 401-409

[3] Tagalakis V, Patenaude V, Kahn SR et al. Incidence of and mortality from venous thromboembolism in a real-world population: the Q-VTE study cohort. Am J Med 2013; 126: 832.e813-821

[4] Kahn SR, Comerota AJ, Cushman M et al. The Postthrombotic Syndrome: Evidence-Based Prevention, Diagnosis, and Treatment Strategies - A Scientific Statement From the American Heart Association. Circulation 2014; 130: 1636-1661

[5] Rabe E, Pannier-Fischer F, Bromen K et al. Bonner Venenstudie der Deutschen Gesellschaft für Phlebologie - Epidemiologische Untersuchung zur Frage der Häufigkeit und Ausprägung von chronischen Venenkrankheiten in der städtischen und ländlichen Wohnbevölkerung. Phlebologie 2003; 32: $1-14$

[6] Prandoni P, Kahn S. Post-thrombotic syndrome: prevalence, prognostication and need for progress. BJH 2009; 145: 286-295

[7] Kahn SR, Ginsberg JS. Relationship Between Deep Venous Thrombosis and the Postthrombotic Syndrome. Arch Intern Med 2004; 164: 17-26

[8] MacDougall DA, Feliu AL, Boccuzzi S] et al. Economic burden of deep-vein thrombosis, pulmonary embolism, and postthrombotic syndrome. Am J Health Syst Pharm 2006; 63: S5-S15
[9] Ginsberg JS, Turkstra F, Buller HR et al. Postthrombotic syndrome after hip or knee arthroplasty: a cross-sectional study. Arch Intern Med 2000; 160 (5): 669-672

[10] Hach W. Das postthrombotische Syndrom, Teil 1: Pathophysiologie. Phlebologie 2010; 39: 28-40

[11] Cogo A, Lensing AW, Prandoni P et al. Distribution of thrombosis in patients with symptomatic deep vein thrombosis. Implications for simplifying the diagnostic process with compression ultrasound. Arch Intern Med 1993; 153 (24): 2777-2780

[12] Johnson BF, Manzo RA, Bergelin RO et al. Relationship between changes in the deep venous system and the development of the postthrombotic syndrome after an acute episode of lower limb deep vein thrombosis: A one- to six-year follow-up. J Vasc Surg 1995; 21: 307-313

[13] Johnson BF, Manzo RA, Bergelin RO et al. The site of residual abnormalities in the leg veins in long-term follow-up after deep vein thrombosis and their relationship to the development of the post-thrombotic syndrome. Int Angiol 1996; 15 (1): 14-19. PMID: 8739531

[14] Amin EE, Bistervels IM, Meijer K et al. Reduced incidence of vein occlusion and postthrombotic syndrome after immediate compression for deep vein thrombosis. Blood 2018; 132 (21): 2298-2304

[15] Wahl U, Grosser V, Ernst $F$ et al. Unfallbedingtes postthrombotisches Syndrom in der ärztlichen Begutachtung. Trauma Berufskrankh 2015; 17: 250-260 https://doi.org/10.1007/s10039-015-0087-4

[16] Kolbach DN, Neumann HAM, Prins MH. Definition of the post-thrombotic syndrome, differences between existing classifications. Eur J Vasc Endovasc Surg 2005; 30: 404-407

[17] Rutherford RB, Padberg FT Jr, Comerota AJ et al. Venous severity scoring: An adjunct to venous outcome assessment. J Vasc Surg 2000; 31 (6): 1307-1312. doi:10.1067/mva.2000.107094. PMID: 10842165

[18] Prandoni P, Lensing AW, Cogo A et al. The long-term clinical course of acute deep venous thrombosis. Ann Intern Med 1996; 125 (1): 1-7. doi:10.7326/0003-4819-125-1-199607010-00001. PMID: 8644983

[19] Brandjes DP, Büller HR, Heijboer $\mathrm{H}$ et al. Randomised trial of effect of compression stockings in patients with symptomatic proximal-vein thrombosis. Lancet 1997; 349: 759-762. doi:10.1016/S01406736(96)12215-7. PMID: 9074574 